\title{
Analysis of the Flow Pipe Arrangement in RTM Process
}

\author{
Jinliang Liu \& Xiaoqing $\mathrm{Wu}$ \\ Key Laboratory for Advanced Textile Composite of Ministry of Education \\ Tianjin Polytechnic University \\ Tianjin 300160, China \\ E-mail: wuxiaoqing@tjpu.edu.cn
}

Tianjin Municipal Science and Technology Commission (NO. 06YFJMJC03100, 08JCZDJC24500)

\begin{abstract}
In RTM process, the condition which the flow of resin in the pipeline according with the Darcy's law is the movement of laminar fluid and the Reynolds number less than 1. This paper simulated the flow of the resin in the pipeline by changing the length and diameter of the passageway of pipeline with finite element analytical method. In the result, the relationship of the fluid speed on the exit in pipeline and Reynolds number, also the scope of flow rate of the resin in the pipes can be gained. The test result shows that: exit velocity had little to do with the length, but the ratio of pipeline diameter. When the diameter of entrance assume value of $6 \mathrm{~mm}, 10 \mathrm{~mm}, 16 \mathrm{~mm}$ and $20 \mathrm{~mm}$ and the diameter of exit $6 \mathrm{~mm}$, $10 \mathrm{~mm}$ and $16 \mathrm{~mm}$ respectively, the maximum speed should be $694.442 \mathrm{~mm}$ per second, $416.667 \mathrm{~mm}$ per second and $260.414 \mathrm{~mm}$ per second accordingly.
\end{abstract}

Keywords: RTM, Reynolds number, Flowing speed of resin, Numerical simulation

\section{Introduction}

RTM (Resin transfer molding) is a composite closed molding technology, which has the advantage of low-cost, high-quality production of composite material (Rudd, C.D., 2004, p1-8) applied to volume production of the small and medium-size components. There are mainly design and manufacture of molding, production and laying of preform, injection, compaction and molding of resin, and it's stripping and post-processing etc (Rudd, C.D., 1997, p. 254-292) in RTM process. The basic method is design and manufacture mold based on product needed, making preform of fiber reinforced materials based on the shape of product needed, and then adding it into mold cavity. After injecting liquid resin into the mold cavity and making preform fully impregnated and cured, and then opens ultimate composite material components. RTM is a semi-mechanized composite molding process, which is suitable for an overall shape, and without secondary shear adhesive (Deng, jie, 2005, p. 50-52). Compare to the traditional process of pressing tank molding or Pultrusion, it saves a lot of tooling equipment, which make the production more efficient, relatively low cost. Through using low-viscosity resin and heating curing process, composite materials are created by the high-quality, low scrap rate, and its production process less depends on the operator's skill level.

RTM process falls under the combined effect of a variety of factors, such as the structure and shape of mold, the material properties of the fibers (permeability (Fan, Xiaoqin, 2006, p. 44-49), interface status), the choice of resin type, and molding process parameters, which molding process is the most important. There are many variables in the mold filling, such as injection pressure, temperature and vacuum injection mouth and the mouth of location, number. It is easy to produce dry patches and molding incomplete phenomenon in the experiment (Wang, Yongxiang, 2005). A computer simulation of the molding process plays a very good role in guiding, thereby it can saves money and shortens the cycle. Due to the importance of RTM process in the industrial application, a good simulation technology develops and the development of several commercial simulation software (Simacek, P., 2004, p. 355-367;Bruschke, M. V., 1990, p. 398-405; Liu, Ningsheng, 2005, p. 40-42; Lu, Mingkun, 2005,p. 31-35), a lot of people simulated and studied mold filling process (Phelan, F. R. Jr., 1992, p. 90-102; Van, Melick, H. G. H, 1998, p. 17-22; Dai, Fuhong, 2004, p. 161-167; Li, Haichen, 2003, p. 58-61; Dai, Fuhong, 2004, p. 335-340; Shojaei,2006, p. 1434-1450; Wang, Baichen, 2006,p. 297-300). In early studies Martin and son (Martin, G. Q., 1986, p. 149-157) simulated the mold filling process, and used the computer software (POLYFLOW) to simulate resin flow. Zhong, Cai (Zhong,Cai, 1992, p. 1310-1338) studied the calculation process of the simplifying RTM resin flow. In view of some simple model, the resin flow of various boundary conditions in entrance can calculate. However, the above-mentioned research work focuses on the totality of the molding resin flow and pressure and speed control, there is no pipeline in the process of the RTM resin flow problems for research. In RTM Process, in a resin preform mold cavity to streamline the flow of fluids in porous media 
flows, and these flows must be consistent with Darcy's law. Before the resin flowing in the pipeline, resin into the mold cavity must also be consistent with Darcy's law, and in order to meet the resin into the mold cavity with Darcy's law, the flow must be laminar flow. This paper uses the finite element method to simulate the resin flow by designing the different length and the diameter rate of the pipeline. With the Darcy's law and the Reynolds number is less than 1, the general law of the resin flow in the pipeline can be obtained.

\section{Theory}

In the RTM process, the resin in the pipeline must be the laminar flow, and the Reynolds number has played a very important role. Reynolds number must be less than 1 which can meet the technical requirement. In order to calculate the flow of resin in the pipeline, the Reynolds number equation as follows:

$$
\operatorname{Re}=\frac{\rho v D_{h}}{\mu}
$$

Where $\rho$ is the fluid density of resin, $\mu$ is the fluid viscosity, $v$ is the fluid velocity and $D_{h}$ is pipe diameter.

In the two-dimensional model, the inside diameter is twice the height of the import. Reynolds number in the calculation of fluid flow is an important role, and by the inertial force and the ratio of viscosity to determine whether the flow is the laminar or turbulent.

In RTM Process, on the assumption that pre-shop preform in the cavity is rigid and the resin is the incompressible Newtonian fluid, and the surface tension is neglected. The interstice of mat is much smaller than the pore size of the cavity, so the resin flow in the closed cavity can be described by the Newtonian fluid flow through porous media. Darcy's law can be expressed as:

$$
v=\frac{K}{\mu} \nabla P
$$

Where $v$ is the resin flow velocity, $\mu$ is the resin viscosity, $K$ is the fiber permeability tensor, and $\nabla \mathrm{P}$ is the pressure gradient.

\section{Calculation}

\subsection{Piping Design}

In the RTM process, the pipeline connects the mold and the resin tank, then resin flows through the pipeline mold cavity. In order to study the impact of pipeline to the process, the design of the pipeline form as shown in Figure 1.

According to different shapes of the pipeline as shown in Figure 2, the import will be divided into length of $50 \mathrm{~mm}$, $100 \mathrm{~mm}, 150 \mathrm{~mm}, 200 \mathrm{~mm}$, radius of $5 \mathrm{~mm}, 8 \mathrm{~mm}, 10 \mathrm{~mm}, 12 \mathrm{~mm}$, and transition of the $20 \mathrm{~mm}$; the export will be divided into length of $20 \mathrm{~mm}, 60 \mathrm{~mm}$, radius of $3 \mathrm{~mm}, 5 \mathrm{~mm}, 8 \mathrm{~mm}$. Import and export pipeline length and radius of a free portfolio, there are a total of 96 combinations.

\subsection{Calculation of simulation process}

In order to better demonstrate the calculation process, the example in view of Fig1is calculated. The import length of the pipeline is $50 \mathrm{~mm}$ with a radius of $5 \mathrm{~mm}$, transition of the $20 \mathrm{~mm}$, and the length of export is $20 \mathrm{~mm}$, with a radius of $3 \mathrm{~mm}$. the flow of resin in the pipeline can be considered as a two-dimensional interface shown in Figure 2 .

In order to study the speed of the export pipeline and pipeline diameter and length how to impact the Reynolds number, assuming that the density and viscosity of the resin is known.

$$
\begin{aligned}
& \rho=1.2 E-3 \mathrm{~g} / \mathrm{mm}^{3} \\
& \mu=\mathbf{0 . 5} \mathrm{g} / \mathrm{mm} \cdot \mathrm{s}
\end{aligned}
$$

Simulation of the process usually consists of three phases: preconditioning, resolution and post-processing. After founding the model shown in Fig2, and separating grid, and setting parameters, ultimately the relationship of the diameter of export pipeline and the speed can be got in Fig3. From the corresponding relations in Fig3 can be seen that in the location of $1.5000 \mathrm{~mm}$, the maximum speed is by $41.939 \mathrm{~mm} / \mathrm{s}$. In the resin exports, in accordance with the maximum speed, into the equation (1) to calculate the Reynolds number, whether to accord with the RTM process. 96 calculations the pace of import and export value of the ratio of the diameter of pipes, pipe diameter can be compared and the proportional relationship between flow rates shown in Figure 4.Relationship:

$$
Y=-\mathbf{0 . 4 7 9 5 3}+\mathbf{2 5 . 6 1 3 5 5} X
$$

With the change of length of import, export and diameter, the speed of flow changes accordingly. From the experimental data, the speed of export-import has to do with ratio of pipeline diameter, and has not to do with the length of the import and export. Under the assumption that the speed of imports is certain number, and the design of the pipe size, when exports diameter of $6 \mathrm{~mm}, 10 \mathrm{~mm}, 16 \mathrm{~mm}$, can be from Fig5, three equations of export speed and the 
Reynolds number are got, as follows:

$$
\begin{aligned}
& Y=\mathbf{2 . 8 5 2 3 9} E-\mathbf{6}+\mathbf{0 . 0 0 1 4 4} X \\
& Y=-\mathbf{1 . 0 5 0 5 5} E-\mathbf{6}+\mathbf{0 . 0 0 2 4} X \\
& Y=\mathbf{7 . 9 7 7 7 7} E-\mathbf{7}+\mathbf{0 . 0 0 3 8 4} X
\end{aligned}
$$

In accordance with the formula (6), (7), (8), and Reynolds number to be less than 1, it can be got several of the largest diameter pipelines at the Reynolds number and the corresponding size. In this way, it can easily chance resin pipes in the future experiment, and better control the resin flow rate.

\section{Conclusion}

In RTM Process, resin flow in the pipeline must be consistent with the Reynolds number $(\operatorname{Re}<1)$, then to conform the requirement to the standard process. By changing the length and diameter of import and export, the speed of the export is simulated and got four equations. Through this four equations, commonly used in pipe size, speed of export and the Reynolds number can be calculated, which ensure compliance with the technical requirements, and the smooth conduct experiments. It is shown that:

(1) Exit velocity had little to do with the length, but the ratio of diameter pipeline.

(2) When the diameter of imports is $6 \mathrm{~mm}, 10 \mathrm{~mm}, 16 \mathrm{~mm}, 20 \mathrm{~mm}$, and if the diameter of export is $6 \mathrm{~mm}$, the speed must be limited range of $0 \sim 694.442 \mathrm{~mm} / \mathrm{s}$; if the diameter of export is $10 \mathrm{~mm}$ and the speed must be limited the scope of $0 \sim$ $416.667 \mathrm{~mm} / \mathrm{s}$; if the diameter of the export is $16 \mathrm{~mm}$ and the speed must be limited the scope of $0 \sim 260.414 \mathrm{~mm} / \mathrm{s}$.

\section{Acknowledgements}

The authors would like to thank the Tianjin Municipal Science and Technology Commission for the sponsorship of this research in providing funding which aided this research.

\section{References}

Bruschke, M. V., Advani, S. G. (1990). Polymer Composite, 11: 398-405.

Dai, Fuhong, Du, Shanyi, Zhang, Boming and Wan, Dianfu. (2004). Journal of Materials Science and Technology, 20:335-340.

Dai, Fuhong, Zhang, Boming and Du, Shanyi. (2004). Journal of Composites, 21:161-167.

Deng, jie (2005). Fibre Composites, 1:50-52.

FAN, Xiaoqin and WU, Xiaoqing. (2006). Fibre Reinforced Plastics/Composites, 3:44-49.

Li, Hai-chen, Wang, Biao and Lin, Xin. (2003). Journal of Harbin institute of Technology, 35:58- 61.

Liu, Ningsheng. (2005). Fibre Composites, 1:40-42.

Lu, Mingkun, Liu, Yang and Zhang, Boming. (2005). Fibre Composites, 2:31-35.

Martin, G. Q., Son, J. S. (1986). Dearborn, 5:149-157.

Phelan, F. R. Jr. (1992). Proceedings of the American Society for Composites, 2:90-102.

Rudd, C.D., Long, A.C., Kendall, K.N. (1997). Cambridge England: Wood head Publishing Limited. pp. $254-292$.

Rudd, C.D., Long, A.C., Kendall, K.N. and Mangin, C.G.E. (2004). Liquid molding technologies. Beijin: Chemical Industry Press.pp.1-8.

Shojaei and Akbar . (2006). Composites Part A: Applied Science and Manufacturing, 37: 1434-1450.

Simacek, P., Advani, S. G. (2004). Polymer Composite, 25:355-367.

Van, Melick, H. G. H., Haagh, G. A. A. V., Van, de. Vosse. F. N., Peijs, T. (1998). Advanced Composites Letters, 7:17-22.

Wang, Baichen, Huang, Yudong and Liu, Li. (2006). Journal of Solid Rocket Technology, 29(4):297-300.

Wang, Yongxiang, Qiu, Guijie (2005). Proceedings of 16th Annual Academic conference on FRP/CM.

Zhong, Cai (1992). Journal of Composite Materials, 26:1310-1338.

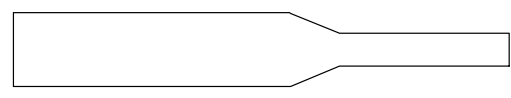

Figure 1. Pipeline form 


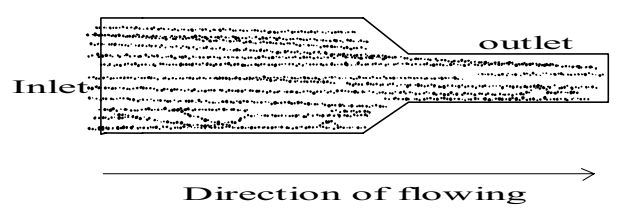

Figure 2. Model of flowing

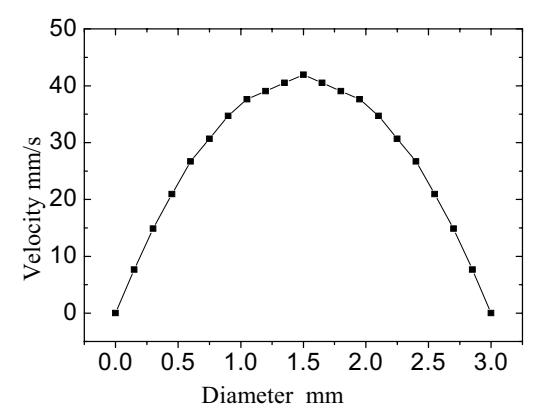

Figure 3. Relationship of diameter and velocity

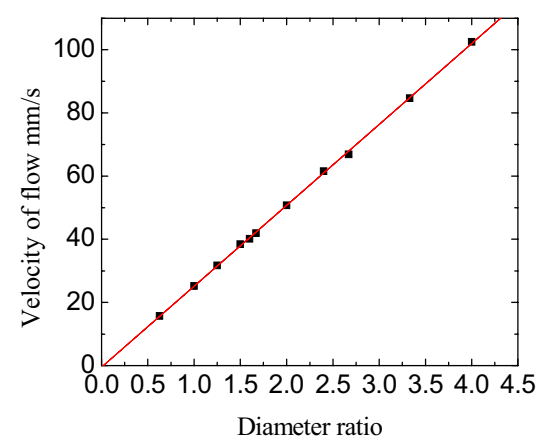

Figure 4. Relationship of diameter ratio and velocity

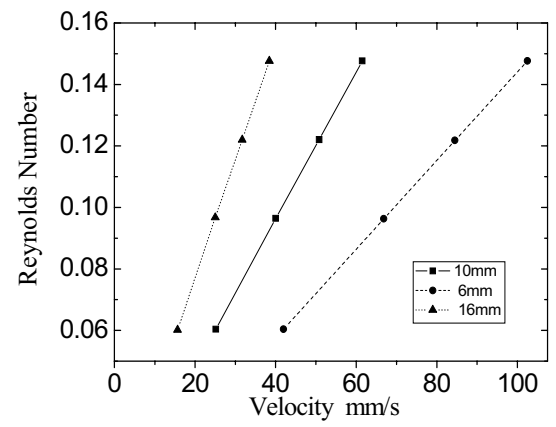

Figure 5. Relationship of exit velocity and Reynolds number 